\title{
Online Surveys Are a Useful Additional Tool in Combination with Clinical Assessments to Easily Assess Demographic and Clinical Data
}

\author{
Stephan Wenninger \\ Department of Neurology, Friedrich-Baur-Institute, Ludwig-Maximilians-University Klinikum Munich, Munich, Germany
}

\section{Dear Editor,}

Please find my response to "Do not replace personal patient/doctor interactions by electronic visits in myotonic dystrophy patients," submitted by Josef Finsterer and Fulvio Alexandre Scorza in European Neurology [1].

I would like to thank Josef Finsterer and his colleague Fulvio Alexandre Scorza for their comments on our publication[2]. They have raised some concerns and brought up considerations, which will be discussed in the following:

- Of course, there is a rough correlation between genotype and phenotype in myotonic dystrophy type 1 (DM1) [3, 4], but - in contrast to the statement by J. Finsterer and F.A. Scorza, this was never described for DM2 $[5,6]$. In DM2, CCTG repeat expansion ranges from approximately 75 to $>11,000$, but the individual repeat size does not correlate with the age of onset or disease severity. The aim of our study was the evaluation of past and present clinical symptoms but not a genotype-phenotype correlation. Therefore, we did not correlate or include genetic test results or medical reports, which was described in the Methods section: "In contrast to other multicenter-based registries, we only focused on the patient's description of their symptom onset, sociodemographic data, and contemporary symptoms and did not include clinical objective data like medical reports (e.g., genetic reports on repeat length) or physical examination results." For the analysis of genotype-phenotype correlations in DM1, several studies have been published, for example, $[3,4,7,8]$.
- Another limitation discussed is bias arising from patients being unable to complete questionnaires due to cognitive impairment or whether the questionnaires could have been completed by their caregivers. Furthermore, in the author's opinion, it remains unclear whether only patients with genetically confirmed myotonic dystrophy or also relatives with suspected DM were included. We have discussed these limitations extensively in our publication, namely, that these online surveys depend on honest and truthful information from patients. Participants were asked at the beginning of the survey (informed consent) whether the questionnaire was completed for oneself or a relative (e.g., a child):

1. I am aged $\geq 18$ years of age and answer the questions by myself (yes/no)

2. If $(1)=$ "no" - I am a parent of my child aged $<13$ and answer the questions on behalf of my child (yes/no)

3. If $(1)=$ "no" and (2) = "no" - I am a child aged 13-17 and answer the questions by myself (yes/no)

4. If $(1)+(2)+(3)=$ "no," stop of questionnaire.

The confirmation of the diagnosis was asked at the beginning of the survey: "We invited adult patients with genetically confirmed DM1 or DM2 who were registered in the German DM registry or the Deutsche Gesellschaft für Muskelkranke e.V. (...) To capture the first symptoms in children without a genetically confirmed diagnosis, we allowed parents with confirmed DMs to fill out the questionnaires for their children, who had a typical clinical

Correspondence to:

Stephan Wenninger, stephan.wenninger@med.uni-muenchen.de 
Table 1. Additional information on the patients who participated in the online survey

\begin{tabular}{|c|c|c|c|}
\hline & DM1 & DM2 & Total \\
\hline Patients $<18$ years of age, $n(\%)$ & $2(0.9)$ & $1(0.5)$ & $3(0.8)$ \\
\hline Patients aged $\geq 18$ years, no genetically confirmed DM diagnosis, $n$ & 0 & 0 & 0 \\
\hline Patients aged $\geq 18$ years, genetically confirmed DM diagnosis, questionnaire answered by caregivers/relatives, $n$ & 0 & 0 & 0 \\
\hline Patients aged $13-17$ years, questions answered by themselves, $n(\%)$ & $4(1.9)$ & $1(0.5)$ & $5(1.3)$ \\
\hline Patients aged $<13$ years, questions answered by their parents*, $n$ & 0 & 0 & 0 \\
\hline
\end{tabular}

* Due to ethical considerations, only patients aged $\geq 13$ years were allowed to fill in their questionnaires by themselves.

presentation of DM" (Methods section). As only patients who were registered in DM patient registries were asked to participate, there should be no patient who participated in this survey without confirmed DM. We have not reported the number of relatives who filled out the questionnaire for their relatives or patients aged $<18$ years without genetic confirmation in detail. Therefore, I have reviewed the raw data again to provide a sufficient answer to this question (Table 1):

Moreover, bias cannot be prevented in any study. For example, a study with personal appointments excludes patients who are not able to travel due to their disability.

In summary, the use of online surveys is easy, convenient, and appropriate from our point of view due the following reasons:

- The number of visits for collecting data on clinical symptoms can be reduced, especially for patients with a severe disease burden. In case severe deterioration is detected by online gathered data, a prompt clinical examination can be scheduled immediately.

- Long and expensive travel, which may be exhausting and demanding, can be avoided. In addition to that, simple follow-up clinical visits can be reduced by online surveys. This decreases the likelihood of missed follow-ups.

- Online surveys can be conducted more frequently than personal appointments and thus provide a more detailed picture of the clinical course.

- Unpredictable events such as the COVID-19 pandemic might also affect data completeness when data are only collected by personal appointments, which might be impossible due to local restrictions.

Furthermore, especially in clinical trials, patients must complete many questionnaires on their clinical status. For most of the questionnaires, it is irrelevant where they are completed. In both methods - face-to-face and online surveys - physicians always rely on the truthful informa- tion provided by patients, which includes the medical history and past and present symptoms. Of course, we could have asked for many more symptoms such as sleep quality and dyspnea, but - as this survey already took $45 \mathrm{~min}$ to complete - we wanted to prevent patients from being overextended. It is always a difficult balance to get as many answers as possible, but also not to overextend patients.

The most important point raised by J. Finsterer is that personal patient-doctor interactions should not be replaced by electronic visits. I fully agree with this statement that physicians should regularly assess patients, which we also emphasized in our publication: "Even if we are aware that online surveys do not replace clinical visits, they might be a valuable additional e-health tool to reduce the number of follow-up examinations and the patient's efforts for sometimes long travels" (Methods section) and "Therefore, we recommend a combination of data collected by online surveys and clinical assessments" and "Nevertheless, as a gold standard, we still recommend a combination of regular clinical assessments and online surveys" (Abstract and Discussion sections). Clinical assessments and online surveys are frequently combined in several clinical trials on neuromuscular disorders, for example, clinicaltrials.gov ID NCT02782741. In addition, assessing symptoms electronically became a useful tool in the last years for many other chronic diseases ("Telemedicine"). The availability of many mobile apps used by clinicians (e.g., for Parkinson's disease, Fabry's disease, migraine, COPD, and many others) reflects the need for an easier way to capture patients' symptoms with neuromuscular diseases in a fast and useful way.

\section{Conflict of Interest Statement}

The author has no conflicts of interest to declare. 


\section{Author Contributions}

S.W. wrote the first draft and final manuscript, and carried out critical revision of the manuscript for intellectual content, discussion, and literature search.

\section{References}

1 Finsterer J, Scorza FA. Do Not Replace Personal Patient/Doctor Interactions by Electronic Visits in Myotonic Dystrophy Patients. Eur Neurol. 2021;1-2.

2 Wenninger S, Stahl K, Montagnese F, Schoser B. Utility and results from a patient-reported online survey in myotonic dystrophies types 1 and 2. Eur Neurol. 2020;83(5):523-33.

3 Overend G, Légaré C, Mathieu J, Bouchard L, Gagnon C, Monckton DG. Allele length of the DMPK CTG repeat is a predictor of progressive myotonic dystrophy type 1 phenotypes. Hum Mol Genet. 2019;28(13):2245-54.

\section{Data Availability Statement}

No data were collected for this response letter. No data are available; no supplementary material is available.
4 Marchini C, Lonigro R, Verriello L, Pellizzari L, Bergonzi P, Damante G. Correlations between individual clinical manifestations and CTG repeat amplification in myotonic dystrophy. Clin Genet. 2000;57(1):74-82.

5 Harley HG, Brook JD, Rundle SA, Crow S, Reardon W, Buckler AJ, et al. Expansion of an unstable DNA region and phenotypic variation in myotonic dystrophy. Nature. 1992; 355(6360):545-6.

6 Yum K, Wang ET, Kalsotra A. Myotonic dystrophy: disease repeat range, penetrance, age of onset, and relationship between repeat size and phenotypes. Curr Opin Genet Dev. 2017; 44:30-7.
7 Okkersen K, Jimenez-Moreno C, Wenninger S, Daidj F, Glennon J, Cumming S, et al. Cognitive behavioural therapy with optional graded exercise therapy in patients with severe fatigue with myotonic dystrophy type 1 : a multicentre, single-blind, randomised trial. Lancet Neurol. 2018;17(8):671-80.

8 Cumming SA, Jimenez-Moreno C, Okkersen $\mathrm{K}$, Wenninger S, Daidj F, Hogarth F, et al. Genetic determinants of disease severity in the myotonic dystrophy type 1 OPTIMISTIC cohort. Neurology. 2019;93(10):e995-1009. 